\title{
Molecular Dynamics Simulation Study of Meso-Confined Propane in $\mathrm{TiO}_{2}$
}

\author{
Siddharth Gautam* and David Cole \\ School of Earth Sciences, The Ohio State University, 275 Mendenhall Laboratory, 125 S Oval \\ Drive, Columbus 43210, Ohio, United States of America
}




\begin{abstract}
We report the structural and dynamical properties of propane confined in mesoporous $\mathrm{TiO}_{2}$, studied using MD simulations. Results indicate that the propane molecules have a tendency to be adsorbed at the pore walls forming sequential layers. Three different regimes of translational motion can be identified which correspond to motion at different time scales accessible to different experimental probes. The variation of the dynamical behavior with loading differs for different regimes and the trend for one of these is consistent with that obtained in recent neutron scattering experiments on propane in mesoporous $\mathrm{TiO}_{2}$ and silica aerogel. The rotational motion of propane molecules confined in the pore exhibits a very fast free rotation at short times which is followed by isotropic rotational diffusion. This work provides an explanation to the counterintuitive observations of an enhancement in the diffusivity with an increased loading of confined propane in recent neutron scattering experiments.
\end{abstract}

Keywords: Molecular dynamics; Mesoporous materials; Diffusion; Rotation

*Corresponding author: gautam.25@osu.edu 


\section{INTRODUCTION}

Most of the materials in the earth crust exhibit a mesoporous nature and play an important role in the flow of energy and matter in the lithosphere [1]. For example, porous rocks are crucial for the migration of hydrocarbons and water through them $[2,3]$. This has resulted in their applications in many industries like gas recovery and catalysis. An important aspect of this applicability is the interaction of the guest species confined in these mesoporous materials amongst themselves and with the confining media [4-6]. A study of the dynamical and structural behavior of these confined gases is imperative in order to understand and exploit the process of gas recovery. Apart from this practical use, a study of the behavior of gases confined in mesoporous materials is interesting as they represent a model system to study the molecular structure and dynamics under confinement. Geometric confinement of materials is known to affect their behavior profoundly and gives rise to peculiar phenomenon like single file diffusion [7, 8], and anomalous dependence of diffusion on the concentration [9-11] as well as on the molecular size of the guest species [12]. These fundamentally interesting features and applications in industries has resulted in a large amount of research devoted to the dynamics of guest species under confinement in microporous [13-24] as well as mesoporous materials [25-33] using various experimental as well as computational tools. Many such studies have been reported in recent reviews [34, 35].

The interaction of a fluid with either a 2D o 3D substrate gives rise to interesting properties at the interface which are absent in the bulk phase [36]. Pore size, shape, connectivity and surface roughness (fractality) may produce somewhat different results compared to a simple 2D surface as pore dimensionality is reduced to the point where the sorption layers start to overlap [37-39]. The interfacial behavior of a weakly wetting fluid like an alkane may experience somewhat different degrees of change in a porous materials compared to a 2D surface if the fluid goes 
through phase change from gas to a liquid to a super critical fluid. In the case of a porous matrices, the curvature of a meniscus may indeed be different compared to what the same fluid experiences on a 2D surface as the fluid experiences condensation.

Mesoporous materials are interesting because they lie intermediate between the strict geometrical confinement imposed by the microporous materials due to reduced pore dimensions on one hand and an absence of confinement in the bulk case at the other extreme. Under confinement by a mesoporous material, a fluid can exhibit interfacial as well as bulk behavior at the same time as has been observed in a recent neutron scattering experiment on ethane dynamics in controlled pore glass [33]. The interfacial effects can be studied by examining closely the population of molecules adsorbed on the pore surface whereas the bulk behavior can be found away from the pore surface. This simultaneous existence of both confined as well as bulk like fluid in the mesopores can give rise to interesting and counter-intuitive dependence of the fluid dynamics on the fluid loading. For example, an enhancement of the diffusivity of the confined fluid with an increase in the fluid loading has been recently observed experimentally [25, 26, 28].

Although a lot of attention has been paid to fluids confined in the microporous materials computationally, studies of fluids confined in mesoporous materials have been relatively few. One difficulty in simulating mesopores is the larger size of the simulation cell that has to be modeled. Recently, however, Mosher et al. have studied methane adsorption in micro and mesoporous carbon systems [40]. Solveyra et al. have simulated structure, dynamics and phase properties of water confined in mesopores of $\mathrm{TiO}_{2}$ [41]. Mesoporous $\mathrm{TiO}_{2}$ is an interesting material due to its applications in energy and environment research especially in the conversion of $\mathrm{CO}_{2}$ into hydrocarbons [42] as well as oxidation of hydrocarbons [43]. Hydrocarbon behavior in $\mathrm{TiO}_{2}$ is therefore interesting from the point of view of these applications too. A widely studied 
hydrocarbon under confinement is propane $[15,16,21-23,25,26]$. Effect of loading on the dynamics of meso-confined propane has also been studied using neutron scattering experiments $[25,26]$. The counterintuitive loading dependence of propane dynamics reported in this study needs further investigation using computer simulations which can reveal properties inaccessible in the neutron experiments. To address these issue concerned with hydrocarbon behavior in $\mathrm{TiO}_{2}$ and its loading dependence, we carried out MD simulation studies of propane confined in $4 \mathrm{~nm}$ mesopore of $\mathrm{TiO}_{2}$. Both structural as well as the dynamical properties of propane were investigated as a function of propane loading in the $\mathrm{TiO}_{2}$ pore. Comparisons of the results obtained are made with studies of propane behavior in other confining media.

\section{SIMULATION DETAILS}

MD simulations reported here were carried out using the DL_POLY software [44]. The calculations were performed using the Deep Carbon Observatory cluster at the Renssaeler Polytechnic Institute, Troy, USA. The simulation box consisted of a rutile $\left(\mathrm{TiO}_{2}\right)$ crystal unit cell replicated $12 \times 12 \times 18$ times in all directions to give a box length of $55.116 \AA$ in the $\mathrm{X}$-Y plane and 53.262A along the Z-direction. To model the pore, atoms from the rutile crystal were etched out in the form of a cylinder $4 \mathrm{~nm}(40 \AA)$ in diameter along the [010] direction. Care was taken to maintain the charge neutrality of the system while etching out the pore. The simulation box with porous $\mathrm{TiO}_{2}$ thus consisted of 8820 atoms. Propane molecules were then loaded in the cylindrical pore. We carried out simulations for three different loadings of propane in the $\mathrm{TiO}_{2}$ pore corresponding to a total number of propane molecules loaded $(\mathrm{N})$ of 224, 112 and 56. Taking into account the volume available in the pores, i.e. the volume of a cylinder with diameter of 40 $\AA$ and a height of $55.116 \AA$, these loadings correspond to a density of $0.237,0.118$ and 0.059 
$\mathrm{g} / \mathrm{cc}$ respectively. These values are smaller than the density of liquid propane at $300 \mathrm{~K}(0.49 \mathrm{~g} / \mathrm{cc}$ at 10 bar) [45]. Propane molecules were modeled in the united atom formalism and consisted of two $\mathrm{CH}_{3}$ sites connected to a central $\mathrm{CH}_{2}$ site with fixed bonds of length $1.54 \AA$ while the angle made by these sites was allowed to be flexible following harmonic motion. TraPPE-UA [46] force field was used to model the interaction between propane molecules.

Interactions of $\mathrm{Ti}$ and $\mathrm{O}$ atoms with the $\mathrm{CH}_{3}$ and $\mathrm{CH}_{2}$ sites constituting propane molecules were modeled with a Lennard-Jones potential. Předota [47] et al. had obtained the Buckingham potentials parameters for $\mathrm{TiO}_{2}$ using ab initio molecular dynamics. Kang et al. [48] used these Buckingham potentials and obtained the Lennard-Jones parameters for $\mathrm{TiO}_{2}$ by empirical fitting. In our simulation we used the potential parameters from refs. 46 and 48 , calculating the crossterms for Lennard-Jones interactions using the Lorentz-Berthelot mixing rules. A complete list of all the interaction parameters is provided in the supplementary material. All Ti and $\mathrm{O}$ atoms were kept rigid throughout the simulation. All simulations were carried out at a temperature of $300 \mathrm{~K}$ in the NVT ensemble employing Nose-Hoover thermostat. Velocity Verlet algorithm was used to advance the positions of the individual interaction sites constituting the molecules and periodic boundary conditions were employed in all directions. This amounts to simulating an infinitely long cylindrical pore in the [010] direction. The distance between the propane molecules in the central pore and those nearest to it in the image pores is larger than the cutoff distance of $14 \AA$ used in the TraPPE-UA force field used here. This excludes all interactions of propane molecules in the central pore and the nearby image pores. A time step of $1 \mathrm{fs}$ was used. An initial simulation of $0.5 \mathrm{~ns}$ was used for the equilibration of the system and trajectories were recorded for calculation of the thermodynamic properties from a simulation run of $1.5 \mathrm{~ns}$ after equilibration. The achievement of equilibrium was confirmed when energy and temperature 
values showed no clear monotonous increase/decrease in time and the variations were less than 5\%. Figure. 1 shows the configuration after equilibration for a loading of 224 propane molecules.

\section{RESULTS}

\section{A. Structure}

\section{Density profile in the pore}

To study the structure of propane confined in the $\mathrm{TiO}_{2}$ nanopore, we calculated its density distribution, using the center of mass to represent the molecule, as a function of the distance from the pore axis, averaged over the entire simulation time. It was normalized by dividing the total number of incidences a molecules is found at a given distance by the product of total number of molecules and the simulation time. Figure 2 shows the normalized density distribution for the three loadings of propane. A large peak centered at $17.5 \AA$ can be seen for all the three loadings studied indicating a tendency of the propane molecules to cluster near the pore wall surface in a layer. The density distribution of the confined propane in the axial direction showed uniform distribution over the entire length of the pore with no heterogeneity. This shows that the peaks in the density distribution in the radial direction represent uniformly distributed molecules in cylindrical shells. In other words these peaks indicate formation of layers. At the lowest loading

almost all the propane molecules occupy the layer that peaks at $17.5 \AA$ from the pore center. As the loading is increased, a second layer represented by a peak at $13.4 \AA$, about a distance of one molecule away from the first peak, appears gradually growing with higher loading. Following this layered structure, we have divided the pore into three different layers. The $1^{\text {st }}$ layer lies between the radial distance $20 \AA$ to $15 \AA$ and contains the first peak completely. All the 
molecules that are outside this layer, i.e. with radial distances smaller than $15 \AA$ form layer 2 . In addition, we assign radial distances between $17.5 \AA$ and $20 \AA$ as forming a layer W. Molecules in this layer lie nearest to the pore wall surface. In what follows we shall analyze the properties of confined propane molecules in these different layers.

The time spent by a typical molecule in a given layer can be quantified by following the time evolution of residence autocorrelation function defined as

$$
R_{i}(t)=\left\langle\theta_{i}(0) \cdot \theta_{i}(t)\right\rangle
$$

where $\theta_{i}$ is a Heaviside step function that takes the value 1 if a molecules is in the layer $i$ at time $t$ and all previous times; and 0 otherwise. The $R_{i}(t)$ functions for the three layers at different propane loadings are shown in Figure 3. An estimate of the time spent by the molecule in the layer can be obtained by approximating the $R_{i}(t)$ functions with exponential decay functions with decay constants giving the average residence times. The residence times obtained thus are given in Table 1. As expected, $R_{i}(t)$ for layer 1 decays the slowest, indicating a large amount of time spent by a typical molecule in this layer. The residence times decrease with an increase in the loading. At the lowest loading, most of the molecules occupy layer 1 and in absence of a second layer there is no exchange between the layers. As the loading is increased, a second layer gradually appears and molecules in this layer are exchanged with those in Layer 1 increasing the outflow of molecules from layer 1, thereby decreasing the residence time. This exchange between the two layers is enhanced further at the highest loading as the second peak becomes more developed. The propane molecules spend least time in Layer 2 in contrast to Layer 1 for the lowest loading because of very low molecular occupancy. The residence time for the layer $\mathrm{W}$ is lowest of all layers for the largest loading. This is because of the small width of this layer. As the 
loading is decreased, the residence times in this layer increase following the trend shown by layer 1 because layer $\mathrm{W}$ is completely contained in layer 1 .

\section{Orientation of propane}

We studied the orientation of a typical propane molecule in the $\mathrm{TiO}_{2}$ pore in the different layers defined above. Figure 4 shows the distribution of propane molecules with respect to the angle made with the pore axial direction and radial direction. For calculating this distribution we have taken the line passing through a $\mathrm{CH}_{2}$ site and the center of mass as a reference. The angle made by this line with the radial and the axial directions is then used to calculate the orientation distributions. The distribution with respect to the axial direction is unimodal. However it gets sharper for the molecules nearest to the wall surface indicating a tendency towards orientational ordering near the surface. This effect is more pronounced in the radial direction, where the distribution functions starts to show a bimodal behavior as the surface is approached.

\section{B. Dynamics}

Dynamical properties of the system can be separated according to the translational, rotational and vibrational degrees of freedom. A separation of the translational and rotational+vibrational motion of a molecule is facilitated by separating the co-ordinates of the constituent sites making a molecule in the space fixed frame and the center of mass frame. The co-ordinates of a representative interaction site constituting the molecule in the space fixed frame of reference $\left(\boldsymbol{r}_{a t}\right)$ can be expressed as a sum of the space fixed co-ordinates of the center of mass of the molecule $\left(\boldsymbol{r}_{\text {СОM }}\right)$ and the co-ordinates of that particular interaction site in the center of mass frame $(\boldsymbol{d})$.

$$
\boldsymbol{r}_{a t}=\boldsymbol{r}_{C O M}+\boldsymbol{d}
$$


The translational motion of the molecule can be tracked by following the space fixed center of mass co-ordinate of the molecule $\left(\boldsymbol{r}_{\mathrm{COM}}\right)$ in time, whereas the co-ordinates of the constituent sites in the center of mass frame $(\boldsymbol{d})$ represent the rotational and vibrational motions. In our simulation, the propane molecules have only one degree of freedom for vibration that is the bending motion, whereas the other vibrational degrees of freedom of bond stretching are constrained. This vibrational degree of freedom can be decoupled from the rotational degree of freedom by following the evolution of a unit vector along $\boldsymbol{d}$ in time. In the subsections below we describe the translational and rotational components of the molecular motion as separated using Eq. 2.

\section{Translation}

The translational diffusive motion of a fluid can be characterized by the mean squared displacement (MSD) of the constituent molecules. The behavior of this quantity in time can indicate the presence of diffusive or sub-diffusive motions. In case of the former MSD varies linearly with time whereas if the MSD variation with time is slower than linear, it indicates a sub-diffusive motion.

Figure 5 shows the variation of MSD of propane in the $\mathrm{TiO}_{2}$ pore with time for the three loadings. The MSD curves show two different slopes with time. For approximately the first 0.5 ps the MSD increases faster with time. This is the ballistic regime covering the time up to which a typical molecule diffuses freely in absence of significant interactions with the neighboring molecules. As the neighboring molecules have no effect during this time, the MSD corresponding to all different loadings is identical. After 0.5 ps the MSD curves for the lowest loadings separate from the rest whereas the two higher loadings are more or less identical. The 
MSD at times larger than $0.5 \mathrm{ps}$ varies linearly with time for all loadings indicating a diffusive motion. Notice that this linearity is not apparent in the main plot which is in the log-log scale. It can be better appreciated in the linear scale plot for one representative loading in the inset of Figure 5. While the MSD curve for the lowest loading shows a slope at long times larger than the rest, indicating a faster diffusion, the slope of the MSD corresponding to the two higher loadings is not very different. We also calculated the MSD components in various directions in the spacefixed as well as the molecular frame of reference. The components of MSD in the directions X, $\mathrm{Y}$ and $\mathrm{Z}$ of the space-fixed frame of reference are shown in the inset of Figure 5. The $\mathrm{Y}$ component of MSD is significantly larger than the $\mathrm{X}$ and $\mathrm{Z}$ components. This is a manifestation of the pore geometry. As the pore axis lies in the [010] direction, there is no spatial restriction on the motion of propane molecules in the $\mathrm{Y}$ directions, whereas rigid geometrical restrictions are imposed by the pore walls in the $\mathrm{X}$ and $\mathrm{Z}$ directions. In addition to this we also calculated the MSD for propane molecules in the direction along and perpendicular to the $\mathrm{CH}_{3}-\mathrm{CH}_{3}$ axis of the molecule which showed no significant difference, indicating the isotropic nature of the diffusion in the molecular frame of reference. Diffusion coefficients can be obtained from the long time slope of the MSD vs time curves. The diffusion coefficient is related to MSD via the Einstein relation;

$$
D=\frac{M S D}{2 n_{d} t}
$$

where $n_{d}$ stands for the number of dimensions of space. Diffusion coefficients calculated from this equation and the MSD curves are given in Table 2. Also given in the table is the ratio of the diffusion coefficient in the Y-direction to the overall diffusion coefficient. This ratio can be seen to increase with a decrease in loading. This is because of a suppression of the diffusivity in the 
$\mathrm{X}-\mathrm{Z}$ plane at lower loading resulting from a decrease in the interlayer exchange of molecules due to the reduction of layer 2 .

Spatio-temporal information about the motion of the molecules can be obtained by studying the intermediate scattering functions (ISF), I(Q,t), representing the molecules. These functions are spatial Fourier transforms of the van- Hove self correlation functions $G(r, t)$, which, in a classical sense stand for the probability that a molecule would be found at position ' $r$ ' at time ' $t$ ' given that the same molecule was at origin at time $\mathrm{t}=0$ [49]. ISFs are measured directly in neutron spin echo measurements, a technique that is used to probe molecular motion in the time scales of a few hundreds of nanoseconds to a few picoseconds range. The temporal Fourier transform of ISFs, dynamic structure factor, $\mathrm{S}(\mathrm{Q}, \omega)$, is also a measurable quantity in neutron scattering experiments and encodes information on the time scales and geometry of the molecular motion.

In an MD simulation, the ISFs can be calculated as

$$
I(Q, t)=\left\langle\exp \left(i \boldsymbol{Q} \cdot\left[\boldsymbol{r}\left(t+t_{0}\right)-\boldsymbol{r}\left(t_{0}\right)\right]\right)\right\rangle
$$

where $\boldsymbol{Q}$ is the momentum transfer, a quantity related to the scattering angle in a scattering experiment and having dimensions of inverse length and $\boldsymbol{r}$ is the position vector of a given interaction site in the simulation. Angular brackets denote an ensemble average. To make the ISF calculated from the simulation comparable to the experiments, an average over all directions of $Q$ vector is taken while calculating the ISFs using Eq. 4. As mentioned above, a separation of translation and rotational motion can be extended to the ISFs, using $\boldsymbol{r}_{\mathrm{COM}}$ in Eq. 4 to get the translational ISF (TISF) and using a unit vector along $\boldsymbol{d}$ to calculate the rotational ISF (RISF).

Usually the TISF exhibits an exponential decay in time, but because of the complexities involved in a molecular ensemble, a single exponential function is sometimes not adequate to describe the 
behavior of a TISF. In Figure. 6 we plot the TISFs for the translational motion of a propane molecule corresponding to five different values of $Q$. Also included is a calculated exponential decay function with a decay constant of 12 ps. It can be seen that the behavior of TISFs calculated from the simulation differs considerably from the exponential decay. The TISF for the smallest $Q$ decays faster at initial times for up to a few picoseconds and then becomes slower at times larger than $10 \mathrm{ps}$. This facilitates the separation of translational motion in different time regimes. At small time scales the motion of a typical propane molecule is fast, but at larger time scales it starts to experience the effects of the presence of other molecules that slows its motion. We found that all TISFs could be modeled by a combination of three exponential decay functions. The decay constants from these, for the two loadings $(\mathrm{N}=224$ and $\mathrm{N}=56)$, are plotted in Figure. 7. The intermediate loading $(\mathrm{N}=112)$ values which lie between the values corresponding to the loadings $\mathrm{N}=224$ and 56 are not shown for the purpose of clarity in representation. More information about the modeling of TISFs is provided in the supplementary material.

It can be seen that the difference between the decay constants corresponding to the two loadings is significant only for the slower components. This is reasonable as the effect of environment is experienced only at larger time scales as mentioned earlier. Moreover, the variation with loading exhibits different behavior for the fast and slower components. While the faster component is seen to exhibit slightly faster dynamics at lower loading, the two slower components exhibit a slowing down of dynamics with a decrease in the loading.

\section{Rotation}


As noted earlier, the rotational and vibrational components of the motion can be separated from the translational component by following the atomic co-ordinates of the molecule in the center of mass frame. We could follow, for example, the evolution of the position vector of the $\mathrm{CH}_{2}$ site in the center of mass frame. To separate the purely rotational component from this, we can follow the evolution of a unit vector directed along the position vector of the $\mathrm{CH}_{2}$ site in the $\mathrm{COM}$ frame $(\boldsymbol{u})$. This unit vector would form a fixed length dipole, the orientational evolution of which would solely come from the rotation of the molecule. We thus calculate the dipole autocorrelation function $C_{R}(t)$ corresponding to the rotational motion as

$$
C_{R}(t)=\langle\boldsymbol{u}(0) \cdot \boldsymbol{u}(t)\rangle
$$

The dipole correlation function can in fact be identified with the second component of a family of functions of Legendre polynomials of the time correlation of unit vector $\boldsymbol{u}, C_{l}=\left\langle P_{l}[\boldsymbol{u}(0) \cdot \boldsymbol{u}(t)]\right\rangle$ which are observed in rotational spectroscopy. While the first component, with $l=0$ is time independent, the second component with $l=1\left(C_{R}(t)\right)$ mentioned above can be measured in dielectric relaxation spectroscopy and the third member with $l=2$ can be measured in light scattering and NMR experiments. In Figure 8 we show the two components of this family with $l=1$ and 2 . In general, these functions are expected to exhibit an exponential decay in case of diffusive rotational motion. However, at very short time scales, in absence of collisions, free rotation of molecules gives rise to a rapid non-exponential decay [50]. In the present case, the switch from free rotation to rotational diffusion occurs at around 0.5 ps as can be seen in the inset of Figure 8. No difference can be seen in the correlation functions for different loadings in this initial part of free rotation as would be expected. As the rotation becomes diffusive due to collisions with neighboring atoms, the fluid loading starts affecting the correlation functions. 
Another interesting feature is the difference in the behavior of these functions in different layers. While in the initial free rotation regime no difference is observed for the molecules in the different layers, the Legendre polynomial functions decay differently for different layers in the diffusive regime. The functions decay slowest for the layer of molecules that are nearest to the wall whereas this decay is much faster for the molecules in the layer 2, away from layer 1 and the pore wall.

The time scale of the rotational motion can be quantified by integrating the $C_{l}(t)$ functions to obtain the rotational correlation times $\tau_{l}$ as

$$
\tau_{l}=\int_{0}^{\infty} C_{l}(t) d t
$$

We used the upper limit of integration as $35 \mathrm{ps}$, long after the correlations decay to zero. The values obtained for $\tau_{1}$ and $\tau_{2}$ corresponding to different loadings are tabulated below. These correlation times would represent average contributions from both the free rotation and the diffusive rotation at longer times.

The quantitative information obtained above for the rotational motion can be supplemented by the information on the geometry of motion derived from the rotational intermediate scattering functions, RISFs. These functions can be used to obtain temporal as well as spatial information on the rotational motion. As noted earlier, these functions can be calculated by replacing $\boldsymbol{r}$ in Eq. 4 by $\boldsymbol{u}$.

The RISFs do not decay to zero because of the spatial restriction inherent in the rotational motion. In COM frame, the atoms of the molecule are not allowed to move to distances larger than the molecular size and so even after long times, some spatial correlation remains. This asymptotic value of the RISFs at long times is identical to the Elastic Incoherent Structure Factor 
(EISF), a quantity that is measured in a quasielastic neutron scattering (QENS) experiment. The variation of this quantity with $Q$ reveals information about the geometry of the rotational motion. The RISFs calculated from the simulation are plotted in Figure 9 whereas Figure 10 shows the EISF extracted from these functions.

In a pore environment, where the geometrical restriction imposed by the confining media is larger than the size of the confined molecule, no preferred orientation of the molecule exists and the molecule performs isotropic rotation. The RISF in such a case is given by

$$
I_{\text {rot }}(Q, t)=\sum_{l=0}^{\infty}(2 l+1) j_{l}^{2}(Q r) \exp \left[-l(l+1) D_{R} t\right]
$$

where $D_{R}$ is the rotational diffusion coefficient and $j_{l}$ are the $l$ th order Bessel functions and $r$ stands for the radius of gyration of the molecule. The first term in this series is a time independent term and is identical to EISF. Thus the EISF for isotropic rotational diffusion is the square of zeroth order Bessel function with argument $Q r$. In Figure 10, the function $j_{l}^{2}(Q r)$ calculated with $r=1$ (magnitude of the unit vector $\boldsymbol{u}$ ) is shown along with the EISF extracted from the simulation data. As can be seen, there is perfect agreement between the EISF from the simulation and the isotropic rotational diffusion model represented by the function $j_{l}^{2}(Q r)$.

Although the EISF is very well described by the isotropic rotational diffusion model, fitting the RISFs with Eq. 7 resulted in poor fits at low times. This is to be expected as the rotation at small times is dominated by free rotation as noted earlier. This free rotation makes the RISFs decay faster than the model function for isotropic rotational diffusion as described by Eq. 7. Thus, no quantitative information on the time scale of the rotational diffusion is obtained from the RISFs, although these functions describe the isotropic nature of rotation. 


\section{DISCUSSION}

The gradual appearance of a second peak in the density distribution plot of Figure 2 with increasing loading suggests that there is a tendency of formation of layers of propane molecules near the pore walls. The effect of increasing propane loading is to build sequential layers starting from the pore wall and moving towards pore axis. In a small angle neutron scattering experiment, Rother et al. have observed a similar layer formation of propane on the pore walls of silica aerogel [51]. This formation of adsorbed layers has important consequences. Firstly, as can be seen in Figure 5, the mean squared displacement corresponding to the two higher loadings is quite similar while that for the lowest loadings exhibits higher values. This could be due to a complete absence of the second layer in the case of lowest loading. Although sparsely populated, the second layer exists in the intermediate loading.

The residence time of a molecule in a given layer depends on the presence of the other layers as can be seen in Figure 3. Molecules in Layer 1 are seen to have the lowest residence time in the case of highest loading. This is because of the presence of a significant second layer. There is a regular exchange of molecules between the two layers. As the loading is decreased the second layer becomes sparsely populated and the residence time of the propane molecules in this layer decreases. The exchange between the two layers is also decreased making molecules in the first layer in intermediate loading spend more time in that layer. As the loading is decreased further, hardly any molecules occupy the second layer in absence of a second layer the molecules spend more time in Layer 1.

The exchange of the molecules between the Layers 1 and 2 has an effect on the anisotropy of diffusion. The diffusion in the $\mathrm{X}-\mathrm{Z}$ plane is restricted due to the geometrical restriction imposed by the pore walls. However, in addition to this geometrical restriction the molecular motion in 
the $\mathrm{X}-\mathrm{Z}$ plane is also restricted due to the preferential occupation of layers by the molecule. In the higher loadings, the presence of a significant Layer 2 facilitates an exchange between the two layers that gives rise to motion with a significant $X-Z$ component, thereby suppressing the anisotropy ratio $\left(D_{y} / D\right)$. At the lowest loading this exchange no longer exists in absence of the second layer and the $\mathrm{X}-\mathrm{Z}$ component of the diffusive motion is suppressed, thus considerably increasing the anisotropy ratio.

In recent neutron scattering experiments performed on hydrocarbons in mesoporous materials [25, 26 and 28], it has been found that motion of the adsorbed hydrocarbon gets faster at higher loadings, which appears contrary to the behavior in bulk. This was explained as a consequence of strong pore wall adsorbate interactions at lower loadings. Indeed, as can be seen from the variation of time constants obtained from the TISFs, out of the three different time scales of motion, the diffusion of propane molecule for two of them become faster at higher loading. The fastest of these different motions corresponds to the initial small time-scale free motion of the molecules before they experience the effects of neighboring molecules in collisions with them and as such exhibits little variation with loading. This extremely fast motion that occurs at subpicosecond time scale is too fast to be detected at the neutron scattering spectrometer used in the studies mentioned above. The intermediate component falls within the range of the instruments used in these studies and it shows a faster diffusion at higher loadings as seen in the experiments. This component would represent the motion of the molecules, once they start colliding with the neighboring molecules. As noted earlier, the gradual appearance of a second layer at higher loadings facilitates an exchange of molecules between the layers, thus enhancing the diffusivity. The tendency of propane molecules to form layers along the walls thus provides an explanation to the loading behavior of diffusion observed in the neutron scattering experiments. The third 
component is very slow and can be detected by an instrument with extremely high resolution like that accessible with a neutron spin echo instrument. We surmise that this component represents the averaged motion of the molecules after several collisions.

The translational diffusion coefficients of propane in $\mathrm{TiO}_{2}$ obtained here from the MSD curves are larger than those obtained for propane in ZSM5 $\left(D=13 \times 10^{-10} \mathrm{~m}^{2} / \mathrm{s}[15], D=23 \times 10^{-10} \mathrm{~m}^{2} / \mathrm{s}\right.$ [16]) and Na-Y zeolites $\left(36 \times 10^{-10} \mathrm{~m}^{2} / \mathrm{s}\right.$ [21]) having micropores of 0.5 and $1.2 \mathrm{~nm}$ respectively. Propane diffusion in single walled nanotubes (SWNT) are however much faster (D values of up to $40000 \times 10^{-10} \mathrm{~m}^{2} / \mathrm{s}$ have been obtained by Hongjun Liu [23]). Gas molecules are known to diffuse faster in SWNTs than in other confining media of comparable pore size [52]. This is attributed to the smooth energy landscape provided by the SWNTs. In mesoporous materials, propane diffusion shows a stronger dependence on loading and at higher loadings is found to have diffusion coefficients in silica aerogel $\left(76 \times 10^{-10} \mathrm{~m}^{2} / \mathrm{s}[25]\right)$ comparable to the present study. In a recent MD simulation study on propane diffusion in silica slit nanopores $(2.8 \mathrm{~nm})$ a much higher value of $2690 \times 10^{-10} \mathrm{~m}^{2} / \mathrm{s}$ has been obtained for in plane diffusion [53]. The shape and size of the nanopores in this study are however quite different from our study and thus a direct comparison is difficult. In a recent neutron scattering experiment on propane in $4 \mathrm{~nm}$ pores of $\mathrm{TiO}_{2}$ we found a diffusion coefficient of $30 \times 10^{-10} \mathrm{~m}^{2} / \mathrm{s}$ at a temperature of $360 \mathrm{~K}$ [26]. However, the loading of propane in the experiment can be determined only qualitatively. Moreover, as noted earlier, the MSD has contributions from motion at all time scales whereas what is measured in the experiments is just the second component in the TISFs. This component encompasses the same time scales seen in the experiment and shows the same trends as a function of loading viz. propane motion gets faster at higher loadings. Schmid et al. have reported a value of diffusion coefficients of bulk propane at elevated pressures [54]. At 250 bar, 
$294 \mathrm{~K}$ they report a diffusion coefficient of $90 \times 10^{-10} \mathrm{~m}^{2} / \mathrm{s}$ for bulk propane. At normal pressure, this value is expected to go up. The diffusivity of propane in the $\mathrm{TiO}_{2}$ pore obtained in our simulation is thus smaller than the value expected for bulk propane. It needs to be emphasized here that although the density of propane in the $\mathrm{TiO}_{2}$ pore is calculated by taking all the pore volume freely available for propane and yields a density less than that of the liquid propane at $300 \mathrm{~K}$, the tendency of the propane molecules to cluster near the pore walls produces a higher local density in the layers, making comparison with the true bulk system difficult.

The rotational motion of propane in $\mathrm{TiO}_{2}$ pore is much faster, compared to that in other confining media. For example Jobic et al. found a rotational diffusion coefficient of $0.33 \times 10^{12} / \mathrm{s}$ for propane in ZSM5 [15] and Mukhopadhyay et al. obtained a value of $1.05 \times 10^{12} / \mathrm{s}$ for propane in Na-Y zeolite [22]. Because of the presence of a fast free rotation at short times we could not obtain the diffusion coefficients, however, a correlation time of less than $0.5 \mathrm{ps}$ indicates a much faster rotation. A major component of this fast rotation is the very fast free rotation exhibited by the molecules at initial $0.5 \mathrm{ps}$ before the typical molecule collides with the neighboring molecules. We found a slight slowing down of the rotational motion at long times with a decrease in loading. A similar effect of loading on the rotation was also observed by Jobic et al. [15]. Because of the large pore size of $\mathrm{TiO}_{2}$, the pore structure imposes no restriction on the rotational motion and as a result the rotation remain isotropic. No oscillatory behavior of the rotational ISFs was found indicating an absence of libration due to geometrical restriction imposed on the guest molecule.

The rotational motion of molecules in different layers is found to differ in the diffusive regime. The rotation of molecules become successively slow for the molecules near to the pore walls. 
This is consistent with the finding of a tendency of orientational ordering in the molecules near the wall.

\section{CONCLUSION}

Results from an MD simulation of propane in $\mathrm{TiO}_{2}$ reported here indicate that the propane molecules have a tendency to be adsorbed at the pore walls forming sequential layers with decreasing density away from the pore wall. This has important consequences on the dynamical properties, both, rotational as well as translational, of propane in $\mathrm{TiO}_{2}$. Counter-intuitive loading dependence of the diffusive motion, as seen in a similar system using neutron scattering, is a result of this layered structuring and its change with loading. The diffusion coefficient obtained for confined propane is less than the bulk value but higher than that under confinement in microporous media. Out of the three regimes of translational motion found for propane in $\mathrm{TiO}_{2}$ the variation of the dynamical behavior with loading in one regime, i.e. enhancement of diffusivity of confined propane with increase in loading, is qualitatively consistent with the variation observed in a neutron scattering experiments on propane dynamics in mesoporous $\mathrm{TiO}_{2}$ and silica aerogel. This is a result of enhancement of the exchange of molecules between different layers at the higher loadings and thus explains the experimental finding. The rotational motion of propane molecules confined in the pore exhibits a very fast free rotation at short times followed by isotropic rotational diffusion at longer times. This initial free rotation makes propane rotation in mesoporous $\mathrm{TiO}_{2}$ faster by almost an order of magnitude than in other media. A 
tendency for orientational ordering in propane molecules near the pore walls makes the rotational diffusion slower near the pore walls.

\section{Acknowledgement}

DL_POLY_4 is a molecular dynamics simulation package written by I.T. Todorov and W. Smith, and has been obtained from STFC's Daresbury Laboratory via the website http://www.ccp5.ac.uk/DL_POLY. We would also like to acknowledge computational support from the Deep Carbon Observatory. Financial support for SG from the US Department of Energy, Office of Basic Sciences, Geosciences Program is gratefully acknowledged. DRC thanks the Sloan Foundation-funded Deep Carbon Observatory for his support.

\section{REFERENCES}

[1] D. R. Cole, E. Mamantov, and G. Rother, "Structure and dynamics of fluids in microporous and mesoporous earth and engineered materials" in Neutron Applications in Earth, Energy and Environmental Sciences edited by L. Liang, R. Rinaldi and H. Schoeber, Springer, 2009, 547570.

[2] Y. Wang, C. Bryan, H. Xu and H. Gao, Geology 31, (2003) 387.

[3] D. R. Cole, M. S. Gruszkiewicz, J. M. Simonson, A. A. Chialvo, and Y. B. Melnichenko, in

R. Wanty and R. Seal, (Eds.) Water-Rock Interaction, Vol. 1, (2004) pp 735.

[4] G. G. Lash and T. Engelder, AAPG Bull. 95, (2011) 61.

[5] N. Snow, Oil Gas J. 109, (2011) 38. 
[6] D. R. Cole, K. Herwig, E. Mamontov, L. Larese, in H-R Wenk (Ed.) Neutron Scattering in Earth Sciences, Rev Mineral Geochem; 63, (2006) pp 313.

[7] J. Karger, Phys. Rev. E, 47, (1993) 1427.

[8] K. Hahn, J. Karger and V. Kukla, Phys. Rev. Lett., 76, (1997) 2762.

[9] S. Y. Bhide and S. Yashonath, J. Chem. Phys., 111, (1999) 1658.

[10] S. Y. Bhide and S. Yashonath, J. Phys. Chem. B, 104, (2000) 2607.

[11] J. Karger and H. Pfeifer, Zeolites, 7, (1987) 90.

[12] P. K. Ghorai, S. Yashonath, P. Demontis and G. B. Suffritti, J. Am. Chem. Soc., 125, (2003)

7116.

[13] R. Bergman and J. Swenson, Nature 403, (2000) 283.

[14] S. Gautam, S. Mitra and R. Mukhopadhyay, Pramana 71, 809 (2008).

[15] H. Jobic, M. Bee and G. J. Kearly Zeolites 12, 146 (1992).

[16] John B. Nicolas, Frans R. Trouw, John E. Mertz, Lennox E. Iton and Anton J. Hopfinger J. Phys. Chem. 97, 4149 (1993)

[17] D. Chakrabarty, S. Gautam, S. Mitra, A. Gil, M. A. Vicente and R. Mukhopadhyay Chem. Phys. Let. 426, (2006) 296.

[18] S. Gautam, S. Mitra, A. Sayeed, S. Yashonath, S. L. Chaplot and R. Mukhopadhyay Chem. Phys. Let. 442, (2007) 311.

[19] S. Gautam, A. K. Tripathi, V. S. Kamble, S. Mitra and R. Mukhopadhyay Pramana 71, (2008) 1153.

[20] V. K. Sharma, S. Gautam, S. Mitra, M. N. Rao, A. K. Tripathi, S. L. Chaplot and R. Mukhopadhyay, J.Phys. Chem. B 113, (2009) 8066. 
[21] S. Mitra, R. Mukhopadhyay, A. Sayeed, S. Yashonath, and S. L. Chaplot, App. Phys. A 74, S1317 (2002); Ahmed Sayeed, S. Mitra, A. V. Anil Kumar, R. Mukhopadhyay, S. Yashonath and S. L. Chaplot, J. Phys. Chem. B 107, 527 (2003)

[22] R. Mukhopadhyay, A. Sayeed, S. Mitra, A.V. Anilkumar, Mala N. Rao, S. Yashonath and S. L. Chaplot Phys. Rev. E 66, 061201 (2002); R. Mukhopadhyay, A. Sayeed, Mala N. Rao, A.V. Anilkumar, S. Mitra, S. Yashonath and S. L. Chaplot, Chem. Phys. 292, 217 (2003)

[23] H. Liu, Phys. Chem. Chem. Phys. 16, 24697 (2014)

[24] V. K. Sharma, S. Gautam, S. Mitra and R. Mukhopadhyay, Z. Phys. Chem. 224, 133 (2010)

[25] S. Gautam, T. Liu, G. Rother, N. Jalarvo, E. Mamontov, S. Welch and D. Cole, AIP Conf.

Proc. 1591, 1353 (2014).

[26] S. Gautam, R. T. Petersen, T. Liu, S. Patankar, N. Grimm, G. Rother, D. Wallacher and D. Cole, ACS Proc. 247 (2014)

[27] S. M. Chathoth, E. Mamontov and Y. B. Melnichenko and M. Zamponi, Micro. Meso. Mater. 132, (2010) 148.

[28] S. M. Chathoth, L. He, E. Mamontov and Y. B. Melnichenko, Micro. Meso. Mater. 148, (2012) 101.

[29] F. Salles, H. Jobic, T. Devic, V. Guillerm, C. Serre, M. M. Koza, G. Ferey, and G. Maurin, J. Phys. Chem. C 117, (2013) 11275.

[30] L. Wasyluk, B. Peplinska, J. Klinowski and S. Jurga, Phys. Chem. Chem. Phys. 4, (2002) 2392.

[31] D. R. Cole, S. Ok, A. Striolo, A. Phan, in R. M. Hazen, A. P. Jones, and J. A. Baross (Eds.) Rev. Min. Geochem. Vol. 75, (2013) pp 495. 
[32] Y. B. Melnichenko, J. Schuller, R. Richert, and B. Ewen and C.-K. Loong, J. Chem. Phys. 103, (1995) 2016.

[33] S. Patankar, S. Gautam, G. Rother, A. Podlesnyak, D. Tomasko and D. Cole, unpublished results.

[34] D. Ruthven Diff. Fund. 2, 77.1 (2005)

[35] J. Karger and R. Valiullin, Chem. Soc. Rev. 42, 4172 (2013).

[36] S. O. Diallo, L. Vlcek, E. Mamontov, J. K. Keum, Jihua Chen, J. S. Hayes Jr., and A. A. Chialvo, Phys Rev E 91, 022124 (2015).

[37] Ariel A. Chialvo, Lukas Vlcek, and Peter T. Cummings, J. Phys. Chem. C 118, 19701 (2014).

[38] Dimitrios Argyris, D. R. Cole, and A. Striolo, ACS Nano 4, 2035 (2010).

[39] Ariel A. Chialvo, Lukas Vlcek, and David R. Cole, J. Phys. Chem. C 116, 13904 (2012).

[40] K. Mosher, J. He, Y. Liu, E. Rupp, J. Wilcox, Int. J. Coal Geo. 109-110, 36 (2013).

[41] E. G. Solveyra, Ezequiel de la Llave, V. Molinero, G. Soler-Illia, and D. A. Scherlis, J. Phys. Chem. C 117, 3330 (2013).

[42] W. Kim, T. Seok and W. Choi, Energy Environ. Sci.5, 6066 (2012).

[43] M. I. Qadir, J. D. Scholten and J. Dupont, J. Mol. Cat. A 383-384, 225 (2014).

[44] I. T. Todorov, W. Smith, K. Trachenko and M. T. Dove, J. Mater. Chem. 16, 1911-1918 (2006).

[45] E.W. Lemmon, M.O. McLinden and D.G. Friend, "Thermophysical Properties of Fluid Systems" in NIST Chemistry WebBook, NIST Standard Reference Database Number 69, Eds. P.J. Linstrom and W.G. Mallard, National Institute of Standards and Technology, Gaithersburg MD, 20899, http://webbook.nist.gov

[46] M. G. Martin, and J. I. Siepmann, J. Phys. Chem. B 102, 2569-2577 (1998). 
[47] M. Předota, A. V. Bandura, P. T. Cummings, J. D. Kubicki, D. J. Wesolowski, A. A. Chialvo, and M. L. Machesky, J. Phys. Chem. B 108, 12049 (2004)

[48] Y. Kang, X. Li, Y. Tu, Q. Wang, and H. Ågren, J. Phys. Chem. C 114, 14496-14502 (2010).

[49] M. Bee, Quasielastic Neutron scattering, Adam Hilger, Bristol (1988).

[50] R. G. Gordon, J. Chem. Phys. 44, 1830 (1966).

[51] G. Rother, Y. Melnichenko, D. Cole, F. Frielinghaus and G. Wignall, J. Phys. Chem. C, 111, 15736 (2007).

[52] A. I. Skoulidas, D. M. Ackerman, J. K. Johnson and D. S. Sholl, Phys. Rev. Lett., 89, 185901 (2002).

[53] Thu Le, Alberto Striolo and David Cole, Chem. Engg. Sc. 121 (2015) 292.

[54] A. Greiner Schmid, S. Wappmann, M. Has, and H.D. Lüdemann, J. Chem. Phys. 94, (1991) 5643. 
TABLE 1. Residence times obtained by fitting the calculated residence correlation functions with exponential decay for different layers and loadings.

\begin{tabular}{|l|l|l|l|}
\hline Loading & Layer 1 & Layer 2 & Layer W \\
\hline 224 & 57.96 & 9.68 & 3.50 \\
\hline 112 & 107.36 & 3.64 & 7.47 \\
\hline 56 & 188.02 & 0.12 & 11.20 \\
\hline
\end{tabular}

TABLE 2 Diffusion Coefficients of propane in $\mathrm{TiO}_{2}$.

\begin{tabular}{|l|l|l|c|}
\hline \multirow{2}{*}{ Loading } & \multicolumn{2}{|c|}{ Diffusion Coefficient $\left(\times 10^{-10} \mathrm{~m}^{2} / \mathrm{s}\right)$} & Anisotropy \\
\cline { 2 - 3 } & Overall & Y-direction $\left(\mathrm{D}_{\mathrm{y}}\right)$ & $\left(\mathrm{D}_{\mathrm{y}} / \mathrm{D}\right)$ \\
\hline 224 & $76.58 \pm 2.1$ & $193.25 \pm 1.0$ & $2.52 \pm 0.07$ \\
\hline 112 & $73.36 \pm 2.0$ & $188.10 \pm 0.9$ & $2.56 \pm 0.07$ \\
\hline 56 & $101.70 \pm 2.2$ & $283.05 \pm 1.0$ & $2.78 \pm 0.06$ \\
\hline
\end{tabular}


TABLE 3 Rotational correlation times of propane in the $\mathrm{TiO}_{2}$ pore.

\begin{tabular}{|c|c|c|c|}
\hline \multicolumn{2}{|c|}{ Loading } & $\tau_{1}(\mathrm{ps})$ & $\tau_{2}(\mathrm{ps})$ \\
\hline \multirow{3}{*}{224} & Overall & 0.28 & 0.21 \\
\cline { 2 - 4 } & Layer W & 0.47 & 0.47 \\
\cline { 2 - 4 } & Layer 1 & 0.31 & 0.24 \\
\cline { 2 - 4 } & Layer 2 & 0.16 & 0.15 \\
\hline \multirow{2}{*}{112} & 0.31 & 0.23 \\
\hline 56 & 0.32 & 0.23 \\
\hline
\end{tabular}

FIGURE CAPTIONS

FIGURE 1. A snapshot of the system after equilibration in the $X-Z$ plane. Small red dots represent oxygen atoms, large light grey circles represent the titanium atoms whereas the propane molecules can be seen as three dark grey circles connected by bonds.

FIGURE 2. Density profile of propane in $\mathrm{TiO}_{2}$ nanopore at the three loadings indicated. Propane molecules have a tendency to form a layer adjacent to the pore wall. An increase in the loading leads to a gradual formation of second layer after the first layer has been saturated. A blown up view of the peak corresponding to this layer is shown in the inset. The center of the pore is located at $r=0$.

FIGURE 3. Normalized residence time correlation functions for Layer 1 (solid lines), Layer 2 (dotted lines), and Layer W (dashed lines) for different loadings of propane.

FIGURE 4. Orientations distribution of the propane molecules in different layers at a loading of 224 molecules with respect to (a) the axial direction, and (b) the radial direction. A description of 
the angles is shown in the panel (c). A line passing through the center of mass and the $\mathrm{CH}_{2}$ site of the propane molecules is taken as reference (line $\mathrm{BC}$ ). The projection of $\mathrm{BC}$ on the $\mathrm{XZ}$ plane is DB. Angle CBA is the angle made with the axial direction whereas angle DBE represents the angle made with the radial direction. A gradual evolution into a bimodal distribution suggests orientational ordering in the layer nearest to the pore wall.

FIGURE 5. Mean Squared Displacement for propane in $\mathrm{TiO}_{2}$ nanopore for different loadings. The inset shows the MSD curves resolved into individual Cartesian directions for the highest loading.

FIGURE 6. Intermediate scattering functions at different $Q$ values indicated for the loading of 224 molecules. An exponential decay function with a decay constant of $12 \mathrm{ps}$ is shown with a solid orange line for behavior comparison. Evidently the TISFs calculated from the simulation deviate from a simple exponential decay, decaying faster initially and slowing down after a few tens of picoseconds.

FIGURE 7. The decay constants obtained by fitting the TISFs calculated from the simulation with a sum of three exponential decay functions. The three decay constants are shown as a function of $Q$ for the two loadings $\mathrm{N}=224$ (solid symbols) and $\mathrm{N}=56$ (open symbols).

FIGURE 8. Dipole correlation functions corresponding to rotation motion of propane molecules as for (a) different loadings and (b) different layers at a loading of $\mathrm{N}=224$. The functions for $l=1$ are shown as solid curves while those for $l=2$ as dotted curves.

FIGURE 9. Intermediate scattering functions for rotational motion at different $Q$ values indicated for the loading of 224 molecules. The rotational ISFs decay to a non-zero value at long 
times which is identical to the elastic incoherent structure factor (EISF) measured in a quasielastic neutron scattering experiment.

FIGURE 10. Elastic Incoherent Structure Factor (EISF) obtained from the rotational intermediate structure factors. The variation for isotropic rotational diffusion model is shown as solid line.

\section{FIGURES}

Figure 1

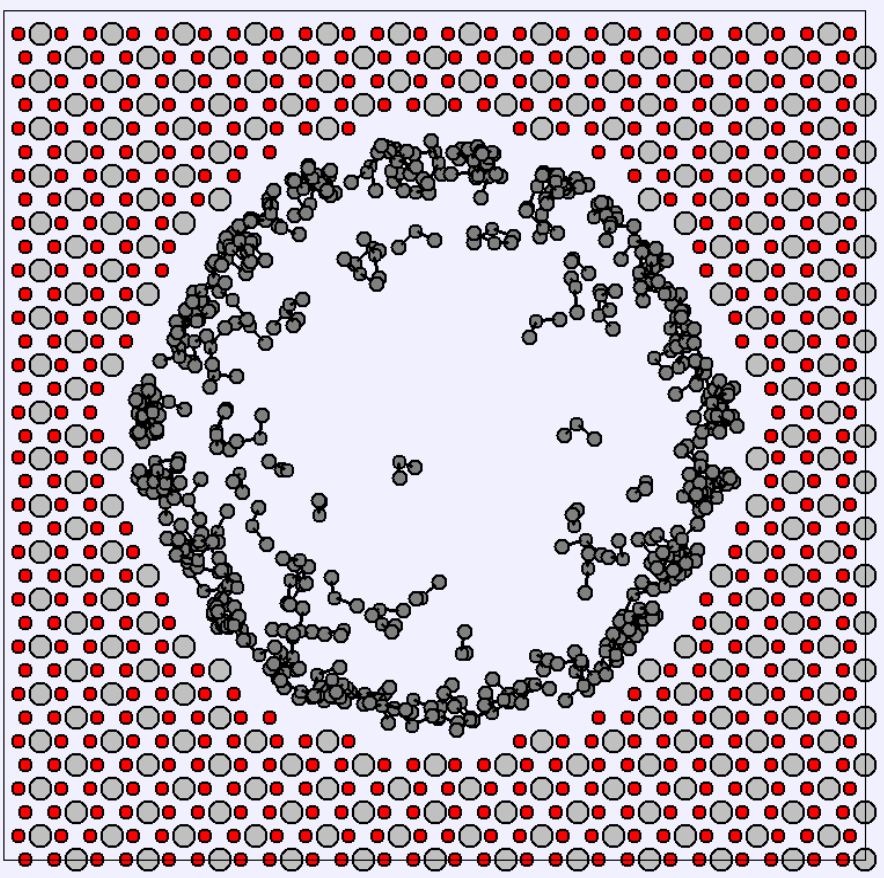


Figure 2

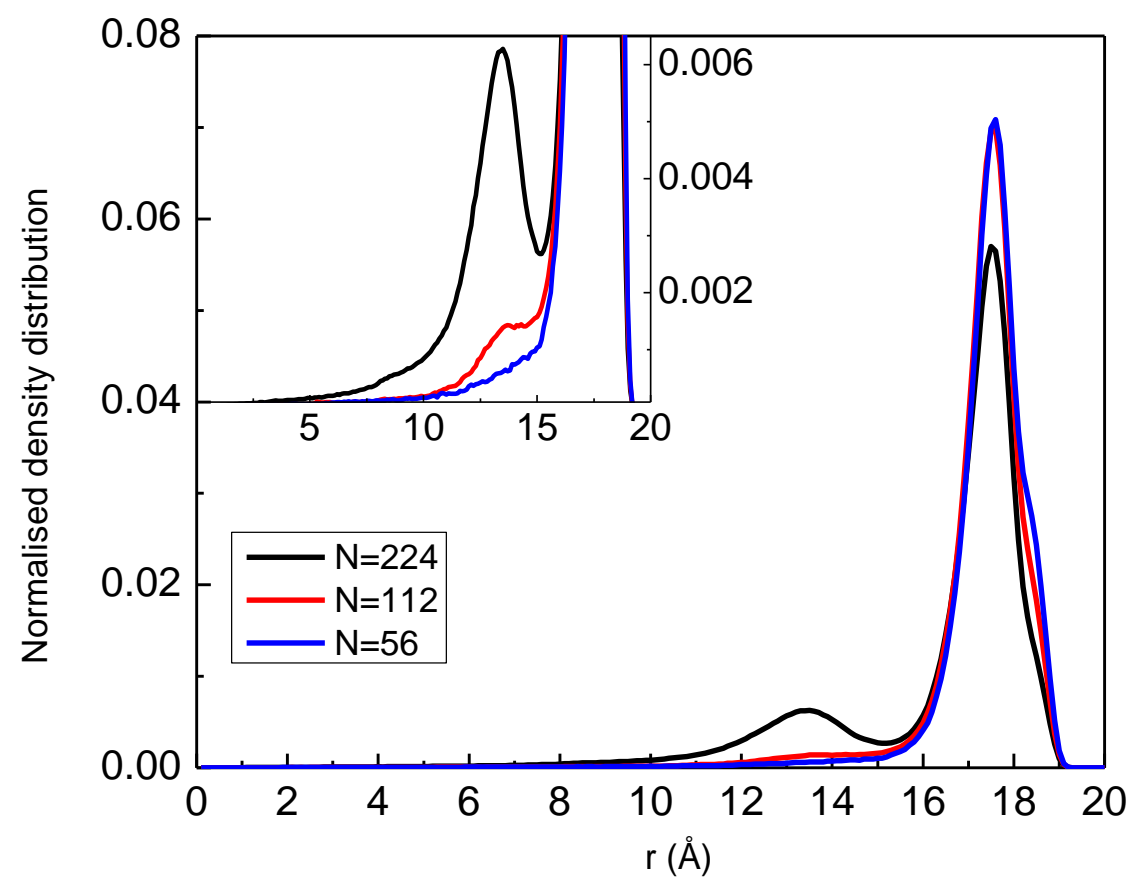

Figure 3

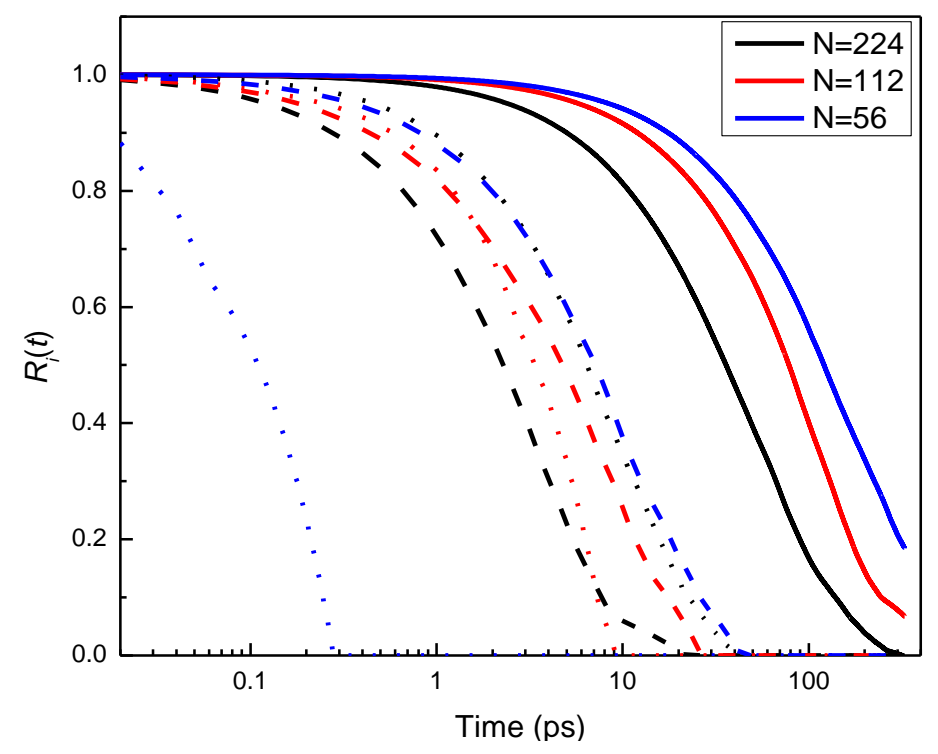

Figure 4 

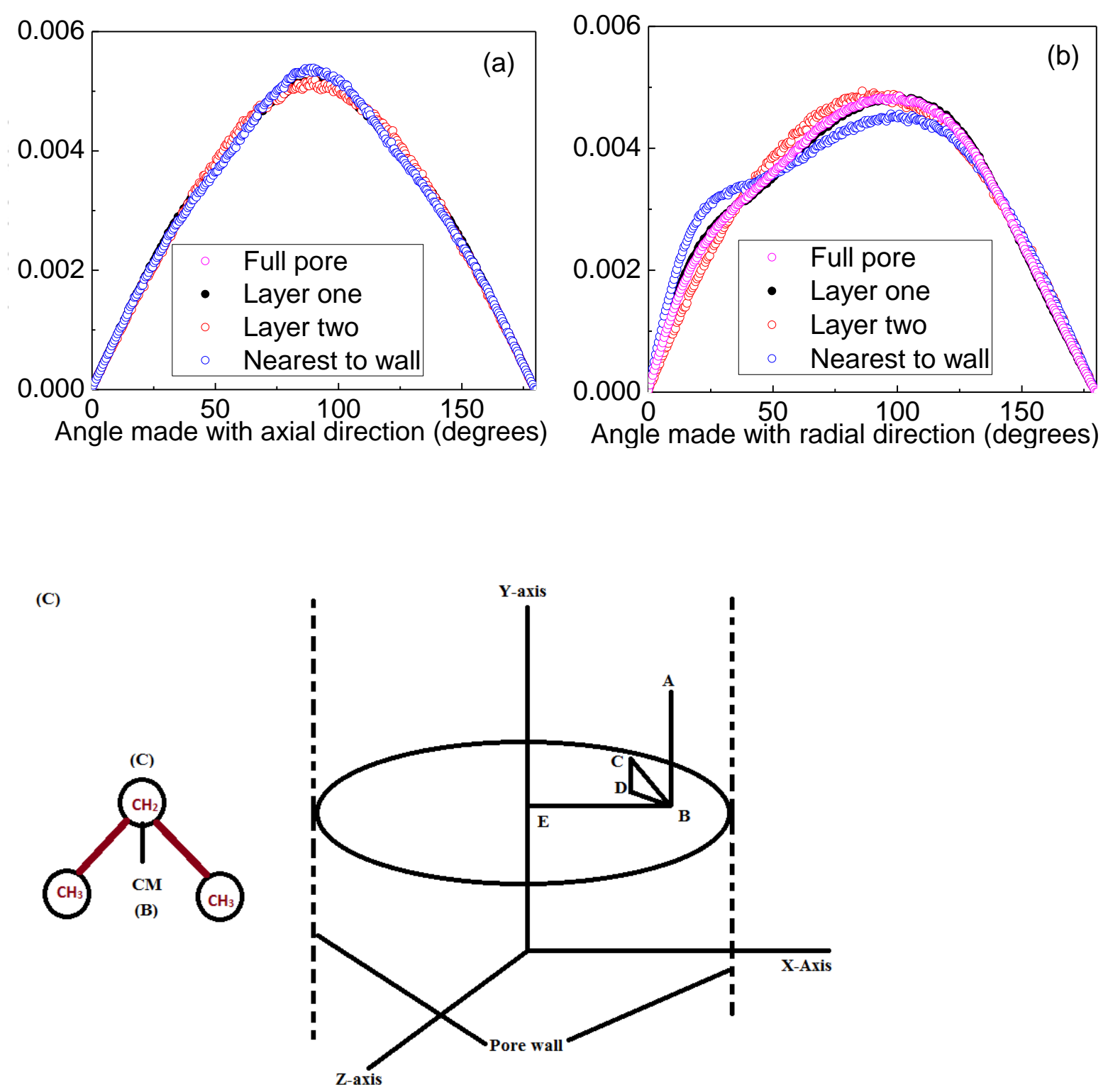

Figure 5 


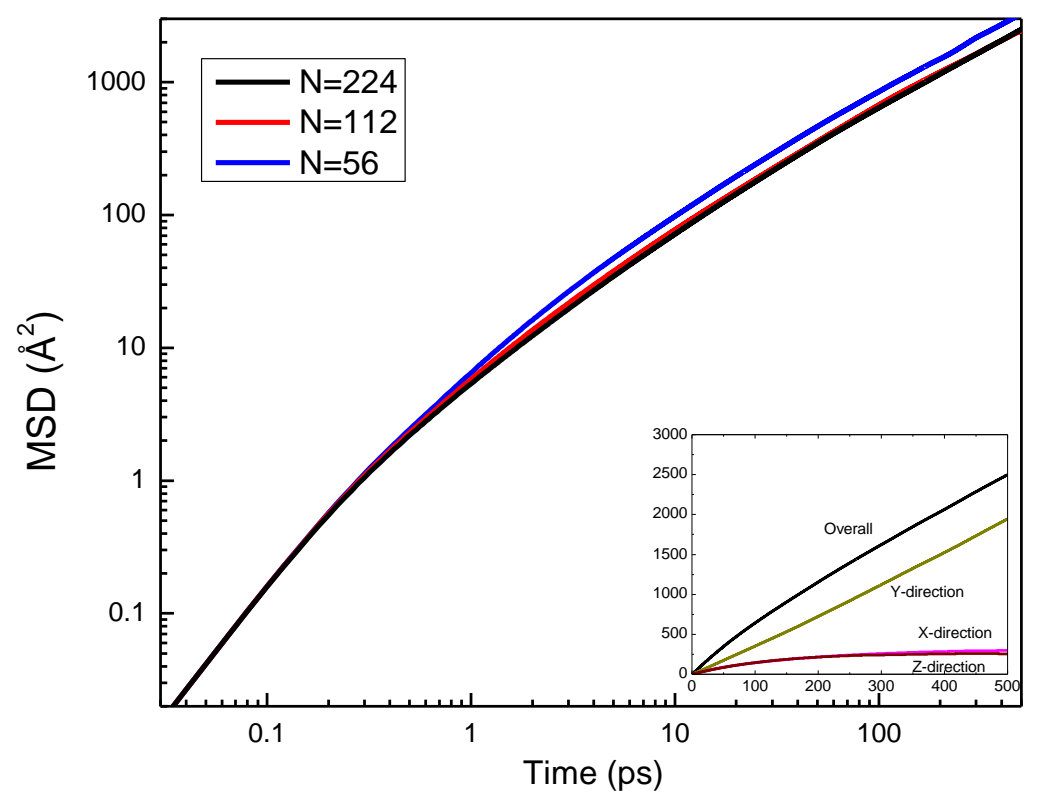

Figure 6 


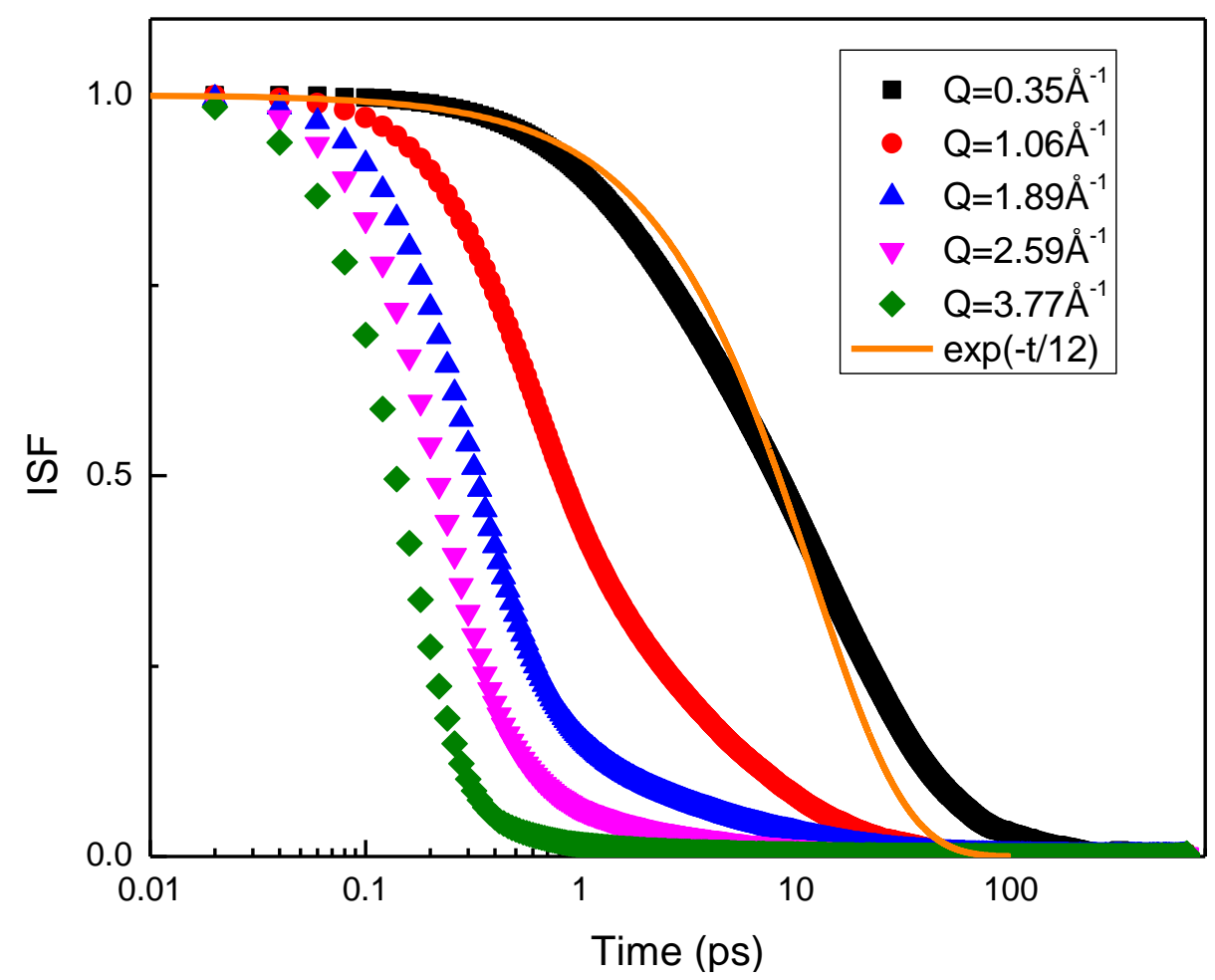

Figure 7

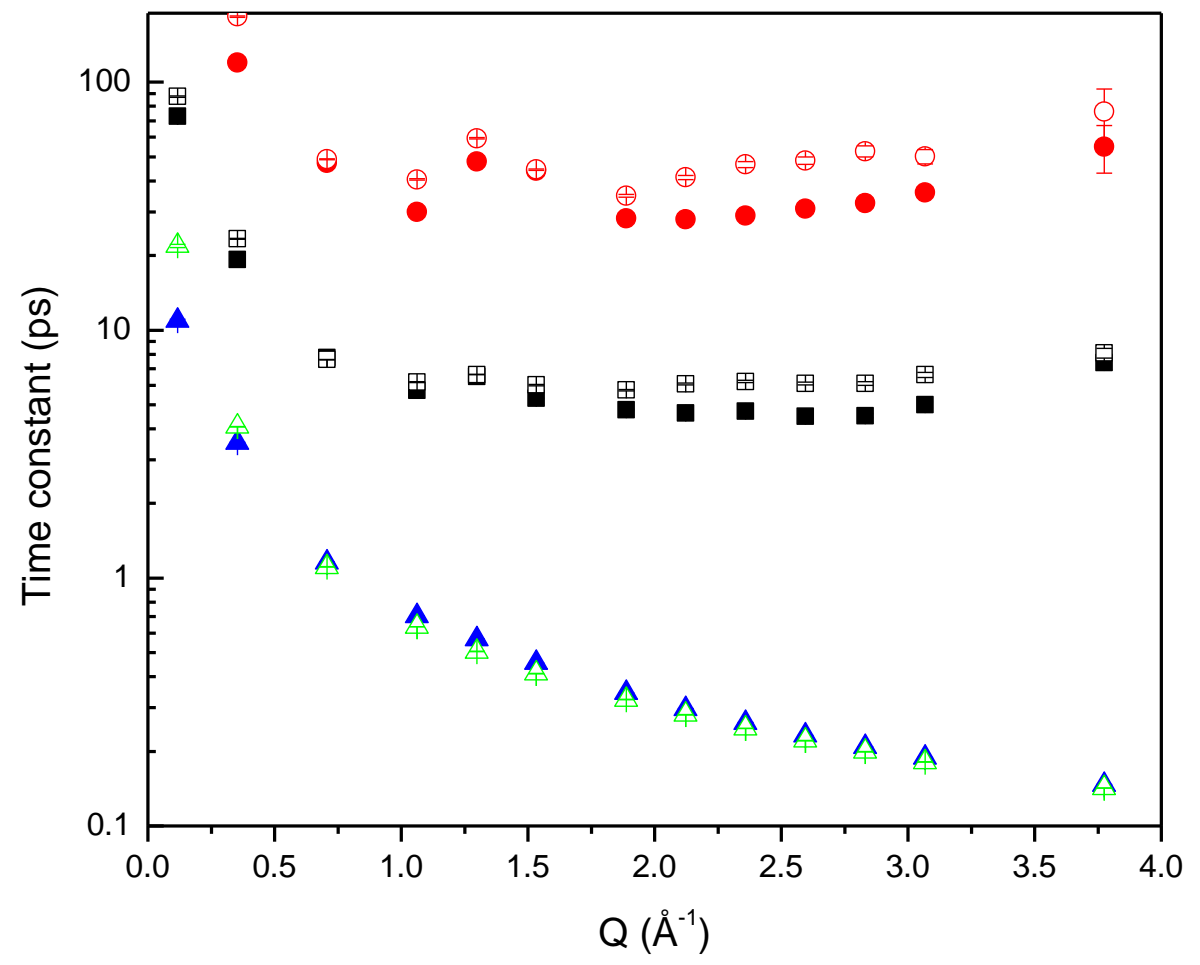

Figure 8 

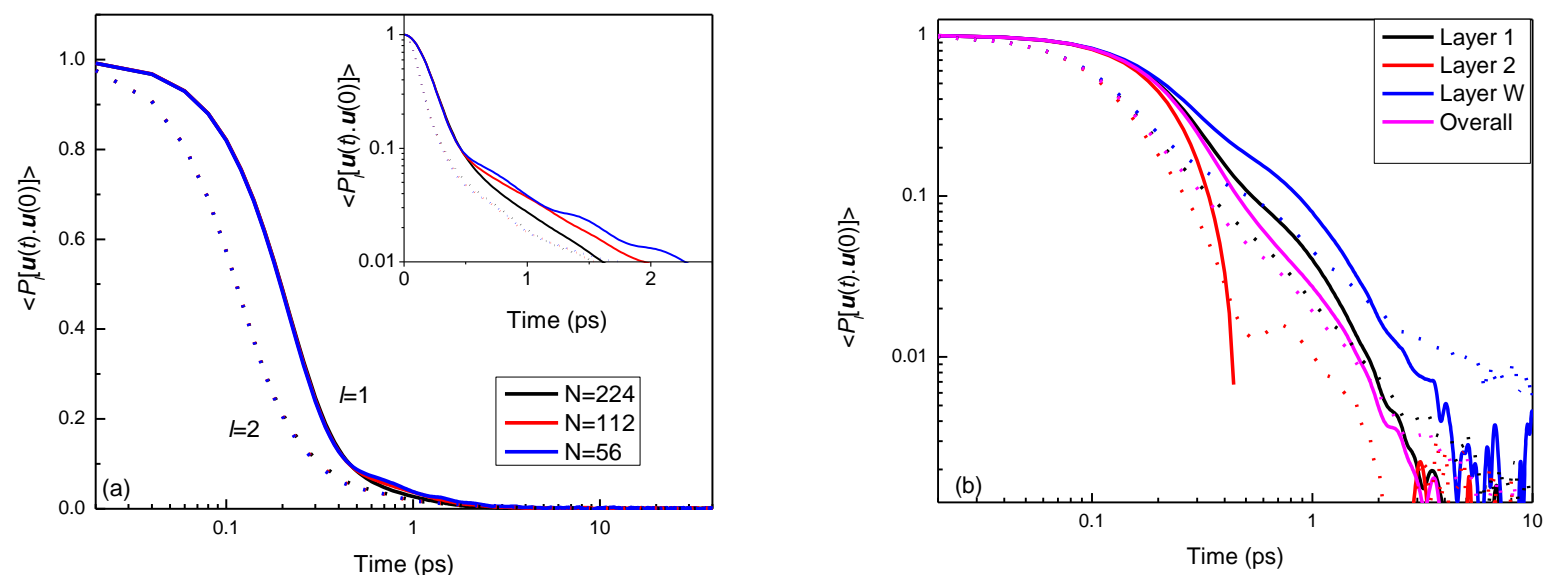

Figure 9

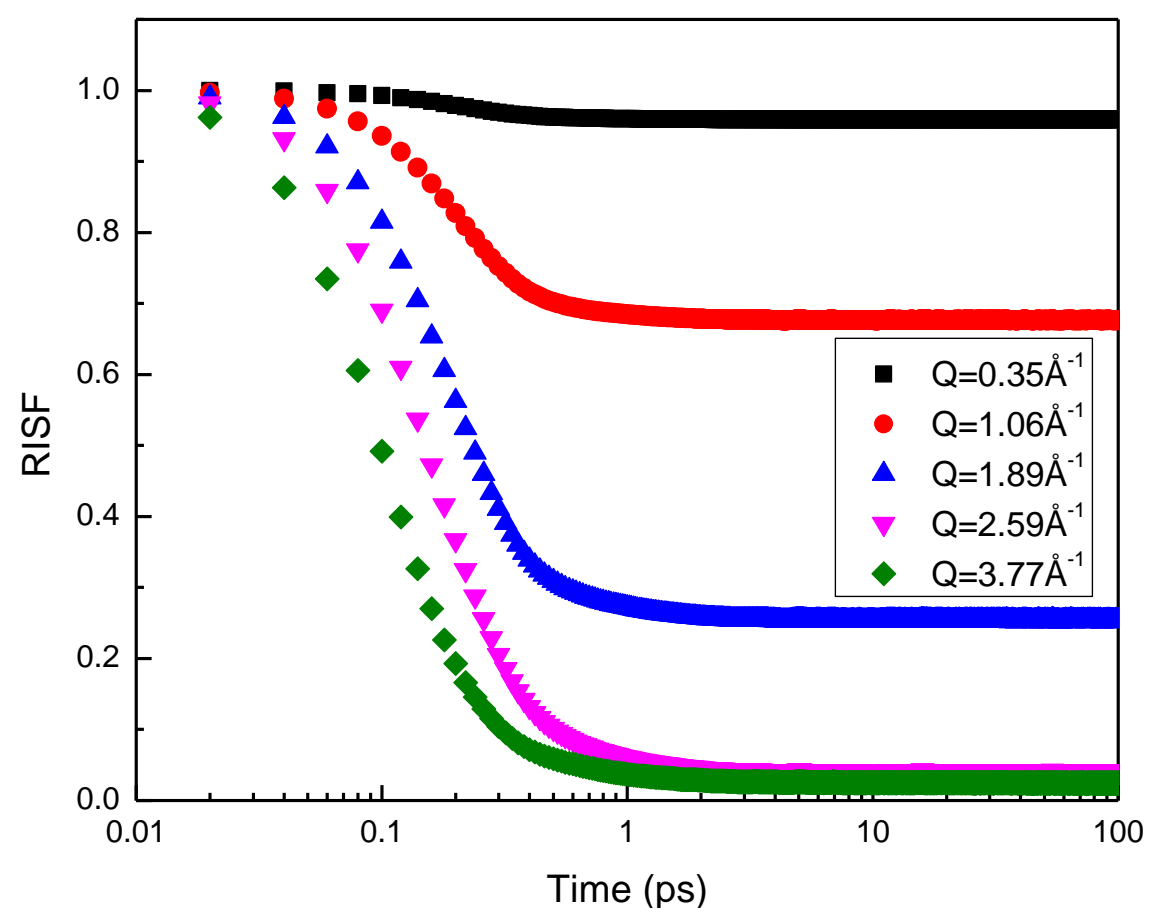


Figure 10

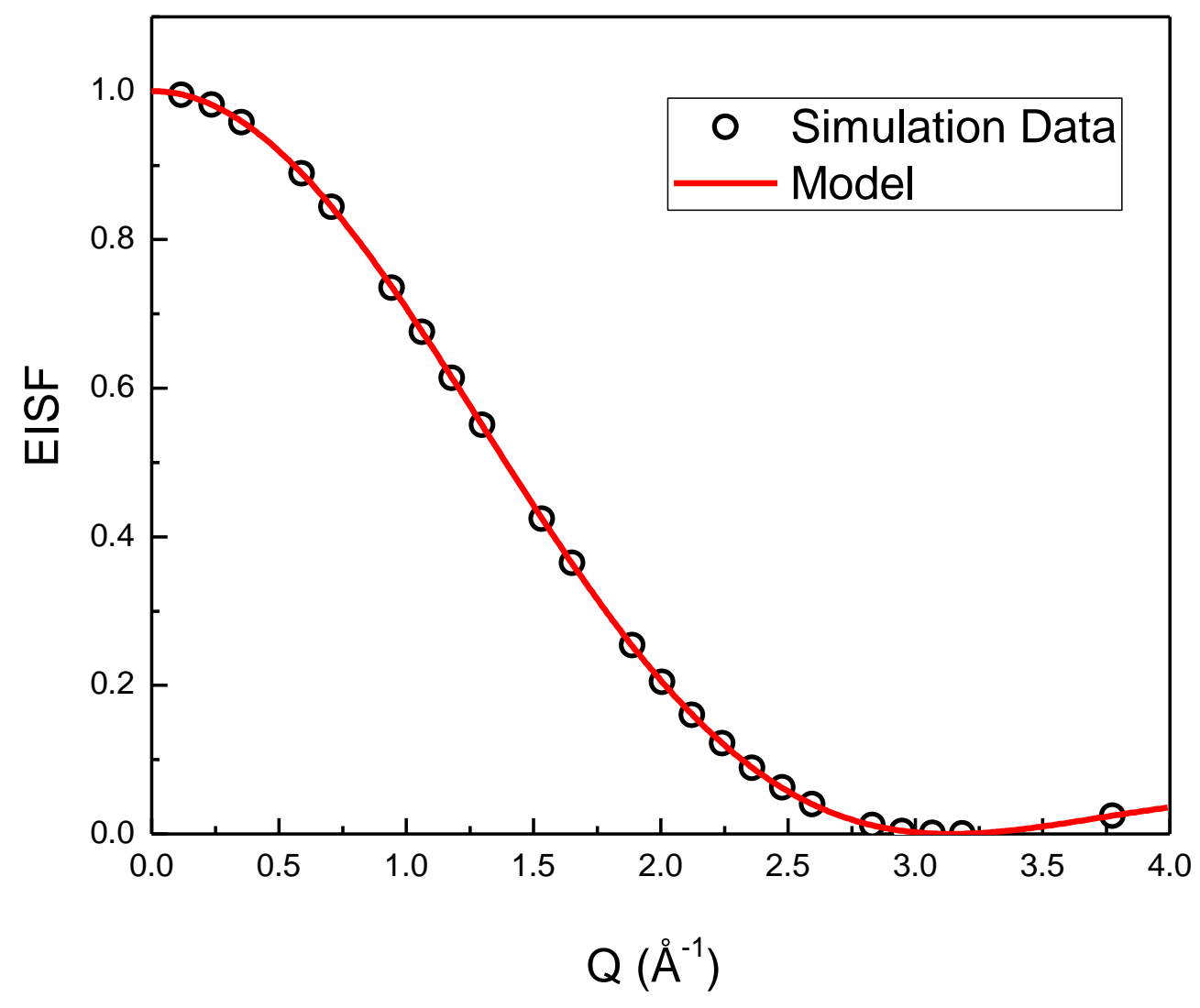




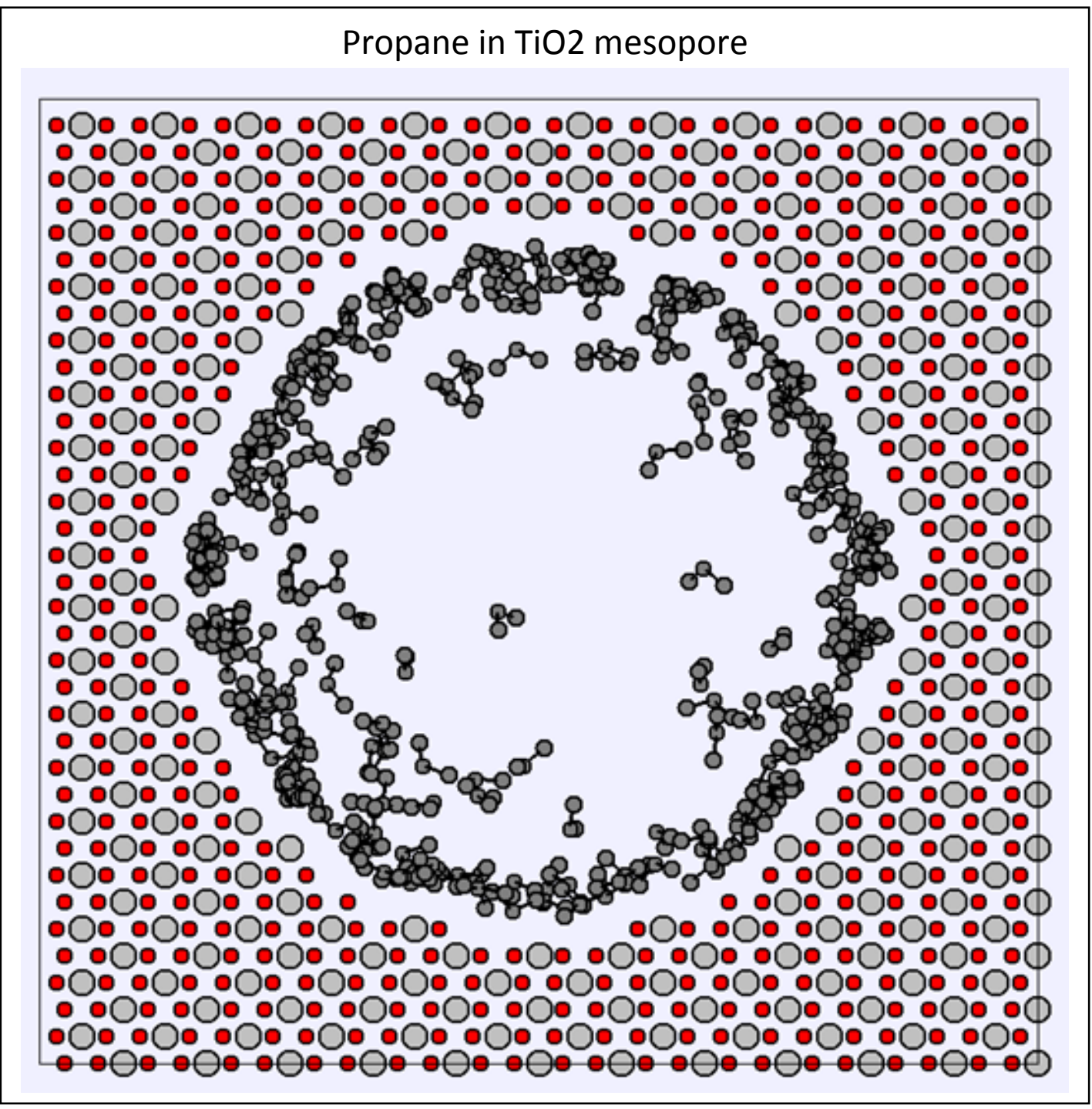




\section{Click here to download Supplementary Materials: Supporting-Info-Gautam-Cole.docx}

Supplementary Materials
Click here to download Su

(2)

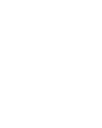
(1)

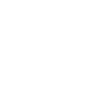

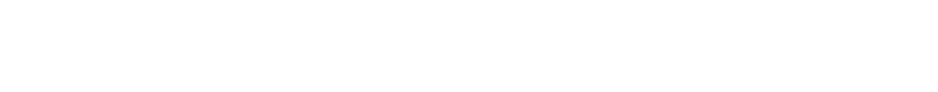
(1) (1) (1)

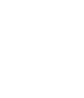

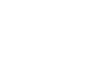

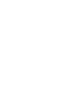
(1)

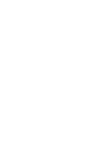
. . .

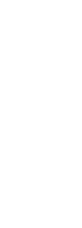
(1) (1) (1) . .

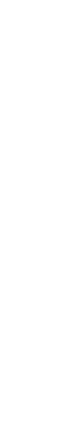

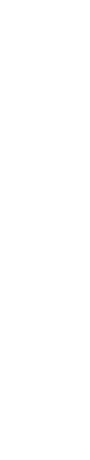

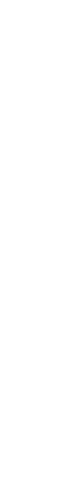

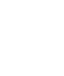

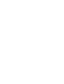
.

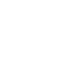
. 